\title{
Caldendrin represses neurite regeneration via a sex-dependent mechanism in sensory
}

neurons

4 Josue A. Lopez ${ }^{1}$, Annamarie Yamamoto ${ }^{1}$, Joseph T. Vecchi ${ }^{1}$, Jussara Hagen, and Amy Lee ${ }^{1,2, *}$

$6{ }^{1}$ Departments of Molecular Physiology and Biophysics, Otolaryngology Head-Neck Surgery,

7 and Neurology, lowa Neuroscience Institute, Pappajohn Biomedical Institute, University of lowa

851 Newton Rd. lowa City, lowa, 52242

$9 \quad{ }^{2}$ Present address: Dept of Neuroscience, University of Texas-Austin

10100 E. $24^{\text {th }}$ St. Austin, TX 78712, USA

11

$12{ }^{*}$ Corresponding author: Amy Lee, 100 E. $24^{\text {th }}$ St. Austin, TX 78712, USA. Email:

13 amy.lee1@austin.utexas.edu

\section{Abbreviations}

16 CaBP1: calcium binding protein 1, DRGN: dorsal root ganglion neuron, KO: knock out, NF200:

17 neurofilament 200, PBS: phosphate buffered saline, PFA: paraformaldehyde, PNS: peripheral

18 nervous system, RAG: regeneration associated gene, WT: wild type.

20 Keywords: Axotomy, calcium, calcium channel, axon regeneration, peripheral nerve injury 
Caldendrin is a calmodulin-like $\mathrm{Ca}^{2+}$ binding protein that is expressed primarily in

29 neurons and regulates multiple effectors including $\mathrm{Ca}_{v} 1$ L-type $\mathrm{Ca}^{2+}$ channels. Here, we

30 tested the hypothesis that caldendrin regulates $\mathrm{Ca}_{\vee} 1$-dependent pathways that repress

31 neurite growth in dorsal root ganglion neurons (DRGNs). By immunofluorescence,

32 caldendrin was localized in medium- and large- diameter DRGNs. Consistent with an

33 inhibitory effect of caldendrin on neurite growth, neurite initiation and growth was

34 enhanced in dissociated DRGNs from caldendrin knockout (KO) mice compared to those

35 from wild type (WT) mice. In an in vitro axotomy assay, caldendrin KO DRGNs grew

36 longer neurites via a mechanism that was more sensitive to inhibitors of transcription as

37 compared to WT DRGNs. Strong depolarization, which normally represses neurite growth

38 through activation of $\mathrm{Ca}_{v} 1$ channels, had no effect on neurite growth in DRGN cultures

39 from female caldendrin KO mice. Remarkably, DRGNs from caldendrin KO males were

40 no different from those of WT males in terms of depolarization-dependent neurite growth

41 repression. We conclude that caldendrin opposes neurite regeneration and growth, and

42 this involves coupling of $\mathrm{Ca}_{v} 1$ channels to growth-inhibitory pathways in DRGNs of

43 females but not males. Our findings suggest that caldendrin $\mathrm{KO}$ mice represent an ideal

44 model in which to interrogate the transcriptional pathways controlling neurite regeneration

45 and how these pathways may differ in males and females. 


\section{INTRODUCTION}

Dorsal root ganglion neurons (DRGNs) are pseudo-unipolar neurons with a peripherally directed neurite that senses information about modalities, such as touch and temperature, which

53 transmits this information into the spinal cord via a centrally directed neurite. In contrast to neurons

54 whose cell bodies reside in the central nervous system, DRGNs retain the ability to regenerate

55 their peripheral neurite following injury (Scheib \& Hoke, 2013). However, the slow rate of regrowth

56 and incomplete re-innervation of target tissues remain significant barriers for therapies to restore

57 sensory function in individuals suffering from peripheral nerve damage (Huebner \& Strittmatter,

58 2009). Despite insights into the molecular mechanisms underlying the functional diversity of

59 DRGNs (Mecklenburg et al., 2020; Wu et al., 2021; Zheng et al., 2019), comparatively little is known about the factors that control the regenerative growth of DRGN neurites.

In many neurons, $\mathrm{Ca}^{2+}$ ions are essential second messengers that can serve as either positive or negative regulators of neurite growth. In cortical and hippocampal neurons of immature

63 rodents, $\mathrm{Ca}^{2+}$ influx through voltage-gated $\mathrm{Ca}_{v} 1$ (L-type) channels promotes the growth and

64 arborization of dendrites via mechanisms that involve calmodulin (CaM)-dependent protein kinase 65 and transcription (i.e., "excitation-transcription" coupling). In the context of DRGNs, however, $66 \mathrm{Ca}_{\vee} 1$ channels trigger pathways that repress neurite regeneration in DRGNs: $\mathrm{Ca}_{\vee} 1$ antagonists

67 facilitate DRGN neurite growth in vitro (Enes et al., 2010; Huebner et al., 2019) and genetic 68 disruption of $\mathrm{Ca}_{\vee} 1$ expression promotes recovery from peripheral nerve injury in vivo (Enes et al., 69 2010). Within DRGNs, the mechanisms whereby Cav1 channels repress neurite growth may 70 involve the transcription of neurite growth inhibiting genes (Enes et al., 2010) or alterations in 71 membrane trafficking and cytoskeletal proteins in the advancing tip of the neurite (i.e., growth 72 cone) (Gomez \& Zheng, 2006; Henley \& Poo, 2004).

73 Caldendrin is a member of a family of $\mathrm{Ca}^{2+}$ binding proteins (CaBPs) that are related to 74 calmodulin (CaM) and highly expressed in neuronal cell-types of the brain, retina, and inner ear 75 (Haeseleer et al., 2004; Laube et al., 2002; Tippens \& Lee, 2007; Yang et al., 2016). In cochlear 
76 spiral ganglion neurons, caldendrin regulates $\mathrm{Ca}_{v} 1$ channels and their coupling to the repression

77 of neurite growth by strong depolarization (T. Yang et al., 2018). To probe the involvement of

78 caldendrin in $\mathrm{Ca}_{v} 1$-dependent mechanisms controlling neurite regeneration, we characterized the

79 expression of caldendrin in DRGNs and analyzed neurite growth in cultured DRGNs from wild-

80 type (WT) and caldendrin knockout (KO) mice. We find that caldendrin is prominently localized in

81 medium- and large- diameter DRGNs and represses neurite regeneration via a transcription-

82 dependent mechanism that involves $\mathrm{Ca}_{v} 1$ channels in females but not in males. Our results reveal

83 an unexpected role for caldendrin in controlling sexually dimorphic pathways converging on neurite growth in sensory neurons.

\section{METHODS}

Animals

All experiments were performed in accordance with guidelines set by the Institutional Animal Care and Use Committee at the University of lowa. The mice (4-8 week old males and females) were housed under a standard 12-h light/dark cycle with access to food and water ad libitum. The

91 generation and characterization of caldendrin KO mice (RRID: MGI: 5780462) was described

92 previously (Kim et al., 2014) and were maintained on a C57BL/6 (Envigo) background.

Immunofluorescence of DRGs

Lumbar DRGs (L4-L6) were harvested from male and female adult WT C57BL/6 mice and immersed in a $4 \%$ paraformaldehyde solution (PFA) made in phosphate-buffered saline (PBS,

97 Cat \#AAJ19943K2, ThermoFisher) and stored at $4{ }^{\circ} \mathrm{C}$ overnight prior to transfer to $15 \%$ sucrose

98 solution in PBS for an additional $24 \mathrm{~h}$. The fixed tissue was molded in optimal cutting temperature

99 compound (Sakura Finetek) and $15-\mu \mathrm{m}$ thick sections were obtained using a cryostat (Leica).

100 Sections were mounted on charged slides and dried on a slide warmer for $5 \mathrm{~min}$. Sections were

101 then subject to rinsing, blocking, and antibody incubations at room temperature (RT). After rinsing 
102 in PBS and incubating in blocking buffer (10\% normal goat serum, Cat \#PCN5000 and $0.2 \%$

103 Triton $^{\mathrm{TM}} \mathrm{X}-100$ Cat \#9002-93-1, ThermoFisher, in PBS) for 30 mins, the sections were incubated

104 for $1 \mathrm{~h}$ in the following primary antibodies diluted 1:1000 in blocking buffer: chicken polyclonal

105 antibody anti-NF200 (RRID: AB_2313552, Aves); chicken polyclonal antibody anti-Peripherin

106 (RRID:AB_10785694, Aves Labs); rabbit polyclonal antibody against caldendrin (UW72)

107 (Haeseleer et al., 2000). Sections were washed 3 times with PBS for 10 min followed by

108 incubation with secondary antibodies diluted 1:500 in blocking buffer: goat anti-Chicken Alexa

109 Fluor®546 (RRID: AB_2534097, ThermoFisher) and Goat anti-Rabbit Alexa Fluor®488

110 (RRID:AB_2534096, ThermoFisher). Sections were then washed 3 times with PBS for 10 min

111 each and mounted using Fluoromount-G® (SouthernBiotech) and allowed to cure for $24 \mathrm{~h}$ in the

112 dark before imaging. DRGs from at least 4 mice were processed in 4 independent experiments.

113 At least 3 sections were analyzed using an Olympus Fluoview 1000 confocal laser scanning 114 microscope with a 20 x lens and FluoView software (RRID: SCR_014215). The numbers of cells 115 exhibiting colocalized caldendrin and NF200 or peripherin fluorescence was determined by 116 researchers blinded to the labeling conditions and genotype.

118 Western Blots

119 Five lumbar WT and caldendrin KO DRGs were harvested from 4-8-week-old male and female 120 mice, flash frozen in liquid nitrogen, and lysed in lysis buffer $(50 \mathrm{mM}$ Tris- $\mathrm{HCl} \mathrm{pH} 7.4,150 \mathrm{mM}$ $121 \mathrm{NaCl}, 0.5 \%$ Triton X100, 0.5\% n-Dodecyl-beta-Maltoside detergent (Thermofisher Cat\# 89902),

122 Protease Inhibitor Cocktail (Roche cOmplete, EDTA-free (Cat\# 05056489001)) and 1 mM PMSF.

123 Plastic pestles were used to homogenize the pellet in a $1.5 \mathrm{ml}$ microfuge tube. Lysates were 124 cleared by spinning down samples at $14 \times 1000 \mathrm{rcf}$ at $4{ }^{\circ} \mathrm{C}$ for $10 \mathrm{~min}$. NuPAGE ${ }^{\text {TM }}$ LDS Sample 125 Buffer (4X) (Cat\# NP0007, ThermoFisher) was added to lysates which were then incubated at 65 $126{ }^{\circ} \mathrm{C}$ for $10 \mathrm{~min}$. The lysate (25\% of total) was loaded into a 4-20\% Tris-Glycine gel (Invitrogen Cat\# 127 XP04200BOX), run in Tris-Glycine SDS Running buffer (Novex Life technologies Cat\# LC2675) 
128 and transferred overnight using Tris-Glycine Transfer Buffer (Novex Life technologies Cat\# 129 LC3675). Blots were incubated in blocking buffer (5\% fat free milk in TBST) for 30 min prior to 130 incubation for $1 \mathrm{~h}$ in primary antibodies diluted in blocking buffer: Goat anti-mouse GAPDH (RRID: 131 AB_1078991, Sigma-Aldrich) 1:1000, goat anti-mouse GAP43 (RRID: AB_2533123, Thermo

132 Fisher Scientific) 1:1000, rabbit polyclonal antibody against caldendrin and goat anti-mouse $\beta$ 133 tubulin (RRID: AB_396360, BD Biosciences) 1:3000. Chemiluminescent detection was 134 accomplished with a Li-COR Odyssey Imager by exposing the blot for 10 min and Image Studio 135 lite was used to prepare images.

DRGN cultures and immunofluorescence

139 DRGN cultures were prepared as previously (Lin \& Chen, 2018). One day before culture 140 preparation, $14 \mathrm{~mm}$ round glass cover slips (Deckglaser) were placed in 24 well plates (Cat\# 141 229123, Celltreat) and coated with poly-L-ornithine $(0.1 \mathrm{mg} / \mathrm{ml}$ in $10 \mathrm{mM}$ borate buffer, $\mathrm{pH} 8.4$, 142 P4957, Sigma) overnight at $37^{\circ} \mathrm{C}$. Coverslips were rinsed with Milli-Q $\mathrm{H}_{2} \mathrm{O}$ three times and coated 143 with laminin $\left(20 \mu \mathrm{g} / \mathrm{ml}\right.$ in ultra-pure $\mathrm{H}_{2} \mathrm{O}, 11243217001$, Sigma) for $1 \mathrm{hr}$ at $37^{\circ} \mathrm{C}$. Mice were 144 anesthetized with isoflurane and subjected to cervical dislocation and decapitation. 20-30 DRGs 145 were harvested into Neurobasal ${ }^{\mathrm{TM}}$ media (Cat\#21103049, ThermoFisher) and DRGNs were 146 dissociated in $0.125 \%$ Trypsin-EDTA (Cat \#25200056, ThermoFisher) and $0.1 \%$ collagenase type 147 I (1 mg/mL in HBSS without calcium or magnesium (-/-)), (Cat \#17100, ThermoFisher) for $40 \mathrm{~min}$. 148 After the enzyme incubation, $10 \%$ horse serum was added to inactivate the proteases, the cell 149 suspension was centrifuged at $500 \mathrm{rcf}$ for $5 \mathrm{~min}$. The neurons were resuspended in Neurobasal ${ }^{\mathrm{TM}}$ 150 media supplemented with 2.5\% L-glutamine (Cat \#25030081, ThermoFisher) and 2\% N-21 (Cat 151 \#SCM081, Millipore Sigma), plated at a density of 5 ganglia per well, and cultured for 18, 24 and $15248 \mathrm{~h}$ in a humidified incubator at $37^{\circ} \mathrm{C}$ with $5 \% \mathrm{CO}_{2}$. In the replating assay (Saijilafu et al., 2013), 153 DRGNs were cultured for $72 \mathrm{~h}$ prior to dissociation with TrypLE ${ }^{\text {TM }}$ Express (Cat\#12604013, 
154 ThermoFisher) for $1 \mathrm{~min}$ and replating and culture for an additional $24 \mathrm{~h}$. Dichlorobenzimidazole

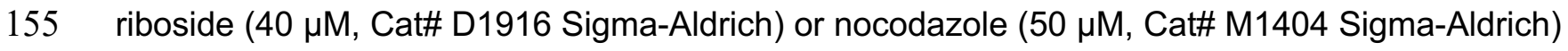
156 diluted in dimethylsulfoxide (DMSO) or DMSO control solution was added to the medium either 157 before or after replating. In some experiments, $40 \mathrm{mM} \mathrm{KCl}\left(\mathrm{K}_{40}\right) \pm$ isradipine (Cat\#2004, Tocris) 158 or nimodipine (each at $10 \mu \mathrm{M})$, Cat\#0600, Tocris)

159 After culture for $18-48 \mathrm{~h}$, media was removed and the coverslips washed with PBS. All subsequent 160 steps were done at room temperature. DRGNs were fixed by adding 4\% PFA for 10 min, washed 161 with PBS (3 times, 5 min each), incubated in blocking buffer for $30 \mathrm{~min}$, and subsequently for $1 \mathrm{~h}$ 162 in chicken polyclonal antibody against NF200 (RRID: AB_2313552, Aves) diluted 1:1000 in 5\% 163 normal goat serum (Cat \#PCN5000, ThermoFisher), 0.5\% TritonTM X-100 (Cat \#9002-93-1, 164 Fisher Scientific), Each well was washed 3 times for 5 mins with PBS followed by addition of 165 Goat anti-Rabbit Alexa Fluor®488 (RRID:AB_2534096, ThermoFisher) (1:1000 in blocking buffer) 166 for $1 \mathrm{~h}$ in the dark. The DRGs were then incubated with DAPI 1:10,000 in PBS for 5 mins. The 167 DRGNs were washed with PBS (3 times, 5 min each). The coverslips were mounted using 168 Fluoromount-G® (SouthernBiotech) and cured for $24 \mathrm{~h}$ in the dark before viewing on an Olympus 169 BX53 microscope with a 20x objective equipped with Olympus DP72 camera and CellSens 170 Standard imaging software (RRID: SCR_014551).

\section{Quantitative analysis of neurite growth}

All quantitative analysis was performed by researchers blinded to experimental conditions.

174 In DRGN cultures, only the NF200 positive neurons with DAPI-positive nuclei were included in 175 the analyses. The \% of DRGNs with neurites was determined by dividing the total number of 176 DRGNs with a neurite at least as long as the diameter of the cell soma with the total number of 177 DRGNs on the coverslip and multiplying by 100. $n=300-400$ neurons per coverslip, 1 coverslip 178 per animal, at least 3 WT or caldendrin KO mice per experiment). For analyses of the longest 
axon, images of DRGNs were acquired using an Olympus DP72 camera and CellSens Standard

180 imaging software (RRID: SCR_014551). The longest axon was measured using Fiji (ImageJ)

181 plug-in NeuronJ(Popko et al., 2009).

182

183 Statistical analysis

184 Statistical analysis was done with Graphpad Prism 9 (RRID: SCR_002798, GraphPad Software,

185 San Diego, CA). Data were first tested for normality by Shapiro- Wilk normality test. If the data

186 were normally distributed, unpaired t-test or ANOVA with Sidak multiple comparison test, with a

187 single pool variance was performed. If data were not normally distributed, Mann-Whitney test was

188 performed. For two-way ANOVA test, with main effects only, and Tukey's multiple comparisons

189 test was performed with individual variances computed for each comparison. Otherwise, post hoc

190 tests were performed, and simple main effects were reported using adjusted $p$ value for multiple

191 comparisons. Data are presented as mean and SEM and $p<0.05$ was considered statistically

192 significant.

\section{RESULTS}

196 Caldendrin is expressed in medium and large diameter DRGNs

197 The CaBP1 subfamily of CaBPs includes caldendrin and the shorter CaBP1 variants

198 (CaBP1-S, CaBP1-L; Fig. 1A) (Haeseleer et al., 2000; Seidenbecher et al., 1998). Caldendrin is

199 the primary CaBP1 variant expressed in the nervous system and exists as $36 \mathrm{kDa}$ and $33 \mathrm{kDa}$

200 forms (Caldendrin-L and Caldendrin-S) that may arise from alternate translational start sites (Kim

201 et al., 2014). By Western blot, only Caldendrin-L and Caldendrin-S were detected with rabbit

202 polyclonal antibodies capable of recognizing all three variants (Kim et al., 2014) (Fig. 1B). The

203 33- and 36-kDa bands likely corresponded to caldendrin since they were detected in DRG lysates

204 from WT but not caldendrin KO mice that lack expression of all 3 CaBP1 variants (Kim et al., 
2014). To gain insights into which subpopulations of DRGNs express caldendrin, we performed double-labeling with antibodies against caldendrin and neurofilament 200 heavy chain (NF200)

207 or peripherin, markers for a marker for myelinated, medium- to large- A- $\beta$ fiber DRGNs and 208 unmyelinated C-fiber and thinly myelinated A-ס fiber DRGNs, respectively (Figs. 1C,D) (Lawson

209 \& Waddell, 1991). Quantitative analyses showed that caldendrin was present in 92\% of NF200-

210 positive neurons and only $\sim 5 \%$ of peripherin-positive neurons. Thus, caldendrin is the major

211 CaBP1 variant expressed in DRGNs and is localized mainly in medium and large-diameter

\section{DRGNs.}

Caldendrin KO DRGNs exhibit enhanced neurite initiation

215 Based on our previous studies of neonatal cochlear spiral ganglion neurons from 216 caldendrin KO mice (T. Yang et al., 2018), we hypothesized that caldendrin acts as a repressor

217 of neurite growth in sensory neurons. If so, then neurite regeneration should be enhanced in 218 DRGNs from caldendrin KO mice as compared to those from WT mice. To test this, we analyzed 219 the percentage of neurons with neurites in cultures of DRGNs from mice. This value was 220 significantly greater in caldendrin KO DRGNs than in WT DRGNs at $18 \mathrm{~h}$ and $24 \mathrm{~h}$, but not at 48 221 h after plating in cultures from male (Fig.2B) and female mice (Fig.2B,C). There was no significant 222 difference in the frequency distribution or median values of neurite lengths in WT and caldendrin $223 \mathrm{KO}$ cultures $24 \mathrm{~h}$ after plating (data not shown). These results suggest that caldendrin represses 224 the mechanism that enables the initial but not later stages of neurite regeneration.

Neurite regeneration is facilitated in caldendrin KO DRGNs following in vitro axotomy

Within $24 \mathrm{~h}$ of dissociation and culture, DRGNs from adult rats develop compact arborizing neurites prior to an elongating, less branched phase of neurite growth by $48 \mathrm{~h}$ (Smith \& Skene,

229 1997). The transition to the elongating growth phase requires gene transcription programs that 230 can be triggered by peripheral nerve injury (Moskowitz et al., 1993; Schreyer \& Skene, 1991) such 
231 that DRGNs cultured from animals subject to in vivo axotomy extend few neurites that rapidly 232 elongate within $24 \mathrm{~h}$ (Smith \& Skene, 1997). To test whether caldendrin influences the arborizing 233 or elongating phase of neurite growth, we used an in vitro axotomy assay in which DRGNs from 234 naive mice are cultured for 3 days prior to replating. The initial 3 days following dissociation mimics 235 the conditioning response to nerve injury that requires gene transcription, such that after replating 236 DRGNs exhibit primarily an elongating pattern of neurite growth that depends on local assembly237 driven mechanisms within growth cones (Saijilafu et al., 2013). If caldendrin represses neurite 238 regeneration following in vitro axotomy, then neurites should be longer in DRGNs from caldendrin 239 KO than from WT mice upon replating. Indeed, the frequency distribution of neurite lengths was 240 shifted rightward, and the median length of the longest neurite was higher in cultures from 241 caldendrin KO than WT mice, regardless of sex (Fig.3A-D). Peripheral nerve injury induces the expression of regeneration-associated genes (RAGs)

243 (Costigan et al., 2002), which is thought to prime DRGNs for the elongating phase of neurite 244 outgrowth (Smith \& Skene, 1997). In the replating assay, RAGs including GAP43 are upregulated 245 in the initial 3-day culture period, similar to that which occurs following peripheral nerve injury 246 (Schreyer \& Skene, 1991). Blunting transcription with the RNA polymerase II inhibitor, 5,6247 dichlorobenzimidazole riboside (DBR) before but not after replating prevents the switch from the 248 arborizing to elongating phase of neurite growth (Fig.4A-C), whereas treatment with the 249 microtubule destabilizer, nocodazole, administered after but not before replating suppresses 250 cytoskeletal mechanisms involved in neurite elongation (Saijilafu et al., 2013). If caldendrin boosts 251 transcriptional alterations that promote neurite regeneration, then DBR should be more efficacious 252 in suppressing neurite outgrowth in caldendrin KO than WT cultures. By contrast, if caldendrin 253 acts solely by the transcription-independent pathway, exposure of the replated DRGNs to ND 254 should more strongly suppress neurite growth in cultures from caldendrin KO than from WT mice.

255 We found that DBR caused a significantly greater reduction in neurite outgrowth in caldendrin KO 256 than in WT cultures, but only when administered during the transcription-dependent phase (i.e., 
257 prior to replating) whereas nocodazole had similar effects in repressing neurite growth in the 258 replated DRGNs from WT and caldendrin KO mice (Fig.4). These results establish that caldendrin 259 represses neurite regeneration and growth via the transcription-dependent rather than the local260 assembly dependent pathway.

GAP43 is the product of a RAG that regulates actin dynamics and facilitates neurite growth during neurodevelopment and regeneration (Chung et al., 2020). By western blot, we noted a

263 time-dependent increase in GAP43 expression and corresponding decrease in caldendrin 264 expression between $3 \mathrm{~h}$ and $3 \mathrm{~d}$ in WT cultures (Fig.5). Thus, downregulation of caldendrin may 265 be necessary for induction of RAGs that support neurite growth.

Sex-dependent role of caldendrin in activity-dependent repression of neurite growth

Although it promotes neurite growth in some neurons (Goldberg et al., 2002; Singh \& Miller, 2005), electrical activity serves as a brake on neurite growth in DRGNs (Fields et al., 1990). through activation of $\mathrm{Ca}_{v} 1$ L-type $\mathrm{Ca}^{2+}$ channels (Enes et al., 2010; Robson \& Burgoyne, 1989).

271 Since caldendrin interacts with and potentiates the opening of $\mathrm{Ca}_{v} 1$ channels (Tippens \& Lee, 272 2007), caldendrin KO DRGNs are predicted to be less sensitive to the growth-inhibitory effects of 273 electrical activity. To test this, we compared the impact of a depolarizing concentration of $\mathrm{K}^{+}(40$ $274 \mathrm{mM}, \mathrm{K}_{40}$ ) on neurite regeneration in WT and caldendrin KO DRGNs. As expected, $\mathrm{K}_{40}$ caused a 275 dramatic reduction in the number of WT DRGNs with neurites at both $18 \mathrm{~h}$ and $24 \mathrm{~h}$ in vitro, and 276 this was abrogated by co-treatment with the $\mathrm{Ca}_{v} 1$ blockers isradipine and nimodipine. In contrast, 277 there was no significant effect of $\mathrm{K}_{40}$ on DRGNs from caldendrin $\mathrm{KO}$ females at either time point 278 (Fig.6A,B). Surprisingly, $\mathrm{K}_{40}$ strongly diminished the number of DRGNs with neurites in cultures 279 from caldendrin KO as well as WT males (Fig.6C). To further probe these sex-specific actions of 280 caldendrin, we compared the effects of $\mathrm{K}_{40}$ administered in the in vitro axotomy assay (Fig.7A).

281 While $\mathrm{K}_{40}$ administered prior to replating strongly inhibited neurite regeneration in a manner that 282 was reversed by nimodipine in cultures from WT females, this effect of $\mathrm{K}_{40}$ was absent in cultures 
283 from caldendrin $\mathrm{KO}$ females (Fig.7B). While $\mathrm{K}_{40}$ reduced neurite lengths when given before but

284 not after replating in cultures from WT females, neurite lengths were not significantly different in

285 cultures from caldendrin $\mathrm{KO}$ females regardless of $\mathrm{K}_{40}$ exposure (Fig.7C). Collectively, our

286 findings suggest that caldendrin couples $\mathrm{Ca}_{v} 1$ channels to activity-dependent transcriptional

287 pathways that repress neurite regeneration in DRGNs in females but not in males.

\section{DISCUSSION}

290 During embryonic development, neurons in the central nervous system extend their axons 291 often over long distances to innervate their targets but later lose this capability, presumably 292 because regenerative signaling pathways become silenced (Yiera \& Bradke, 2006). In DRGNs, 293 severing the peripheral neurite can re-activate the genetic program that promotes growth of both 294 the peripherally and centrally directed neurite (Smith \& Skene, 1997). Thus, DRGNs represent an excellent model in which to study the mechanisms that normally suppress neurite regeneration. Upon dissociation from adult animals, these neurons readily regrow neurites within hours in culture. Our findings that, compared to DRGNs from WT mice, those from caldendrin KO mice had more robustly regenerate their neurites in standard cultures (Fig.2) and following in vitro axotomy (Fig.3) implicate caldendrin in the pathway that opposes the regenerative neurite growth 300 in these primary sensory neurons. Like CaM, caldendrin can bind to and regulate a variety of effector molecules that regulate

$302 \mathrm{Ca}^{2+}$ signaling pathways that impinge on neurite growth dynamics. A major candidate in this 303 regard is the $\mathrm{Ca}_{\mathrm{v}} 1$ channel, which mediates the inhibitory effects of electrical activity on neurite 304 growth (Enes et al., 2010; Robson \& Burgoyne, 1989). Caldendrin and shorter CaBP1 variants 305 bind to the pore-forming subunit of the major $\mathrm{Ca}_{v} 1$ subtypes expressed in the nervous system, $306 \mathrm{Ca}_{\vee} 1.2$ and $\mathrm{Ca}_{\vee} 1.3$, and this interaction potentiates channel opening (Cui et al., 2007; Tippens \& 307 Lee, 2007; Zhou et al., 2004; Zhou et al., 2005). Thus, $\mathrm{Ca}_{v} 1$-dependent $\mathrm{Ca}^{2+}$ signals are expected 308 to be abnormally low in the absence of caldendrin, which we have previously shown to be the 
case in cochlear spiral ganglion neurons of neonatal caldendrin KO mice (T. Yang et al., 2018).

310 If $\mathrm{Ca}_{v} 1$ were similarly depressed in caldendrin $\mathrm{KO}$ DRGNs, $\mathrm{Ca}_{v} 1 \mathrm{Ca}^{2+}$ signals may be insufficient

311 to oppose the transcription of RAGs that such as GAP43. In this regard, it is noteworthy that

312 caldendrin levels were low in WT DRGNs at timepoints when GAP43 was high (Fig.5). Our

313 findings show DBR had a stronger effect in repressing neurite growth in caldendrin KO than in

314 WT cultures (Fig.4) supports the existence of heightened pro-regenerative transcriptional activity

315 in caldendrin KO DRGNs. We speculate that caldendrin controls the activity/expression of

316 transcriptional repressors such as RE1 Silencing Transcription Factor (REST), which suppress

317 transcription of genes that promote axon outgrowth (Oh et al., 2018). In hippocampal neurons,

318 caldendrin binds to and regulates the nuclear localization of Jacob, a protein that controls cAMP-

319 response element binding protein (CREB)- dependent synaptic remodeling (Dieterich et al.,

320 2008). Interactions between CREB and REST pathways have been reported in neuronal gene

321 regulation networks $(\mathrm{Wu} \& \mathrm{Xie}, 2006)$ but whether these interactions occur in DRGNs has not

322 been investigated.

323 In DRGN cultures, the involvement of $\mathrm{Ca}_{v} 1 \mathrm{Ca}^{2+}$ signals in neurite growth is readily 324 revealed by depolarizing concentrations of $\mathrm{K}^{+}$which repress neurite growth in a manner that is 325 reversed by $\mathrm{Ca}_{\sqrt{ }} 1$ blockers (Enes et al., 2010; Robson \& Burgoyne, 1989). The absence of any 326 effect of $\mathrm{K}_{40}$ on neurite growth in caldendrin $\mathrm{KO}$ DRGNs in conventional culture (Fig.6A) and 327 following in vitro axotomy (Fig.7) indicates a critical role for caldendrin in coupling $\mathrm{Ca}_{\mathrm{v}} 1$ channels 328 to activity-dependent neurite growth repression (Fig.6A). The fact that this was only applicable to 329 cultures from female and not male caldendrin KO mice (Fig.6B) was intriguing considering that 330 sex differences have been noted in the transcriptome of mouse DRGNs (Mecklenburg et al., 3312020 ) and in the responses to peripheral nerve injury in animals and humans (Chernov et al., 332 2020; Franco-Enzastiga et al., 2021; Juarez et al., 2019). This result was not due to major 333 differences in caldendrin protein levels in WT males and females (Fig.1B). One possibility is that 334 circulating sex steroids in female mice such as estradiol somehow promote caldendrin 
335 interactions with $\mathrm{Ca}_{\vee} 1$ channels. Estradiol has cell type-specific modulatory effects on $\mathrm{Ca}_{\vee} 1$

336 channels (Lai et al., 2019; Sarkar et al., 2008; Vega-Vela et al., 2017; X. Yang et al., 2018) and

337 so could modify channel gating mechanisms that are targeted by caldendrin.

338 An important follow-up question is how does caldendrin regulate neurite growth in DRGNs

339 from males if $\mathrm{Ca}_{v} 1$ channels are not involved? Like the shorter CaBP1 variants, caldendrin could 340 bind to and inhibit inositol 1,4,5 trisphosphate receptors (IP ${ }_{3}$ Rs) (Haynes et al., 2004; Kasri et al., 341 2004) and therefore $\mathrm{IP}_{3} \mathrm{R}$-mediated $\mathrm{Ca}^{2+}$ signals that are known to enhance DRGN neurite 342 outgrowth (Takei et al., 1998). Alternatively, caldendrin might interact with the actin-binding 343 protein cortactin (Mikhaylova et al., 2018) and A-kinase anchoring proteins (AKAPs) (Gorny et al., 344 2012), both of which have been implicated in neurite growth regulation (Boczek et al., 2021; Kubo 345 et al., 2015).

In summary, we have established a novel role for caldendrin in a sexually dimorphic

347 signaling pathway that represses neurite regeneration in DRGNs in vitro. Establishing the 348 significance of this pathway in peripheral nerve injury models in vivo, as well as an understanding 349 of the mechanisms differentiating this pathway in males and females, remain important challenges 350 for future studies. 


\section{Figure 1}

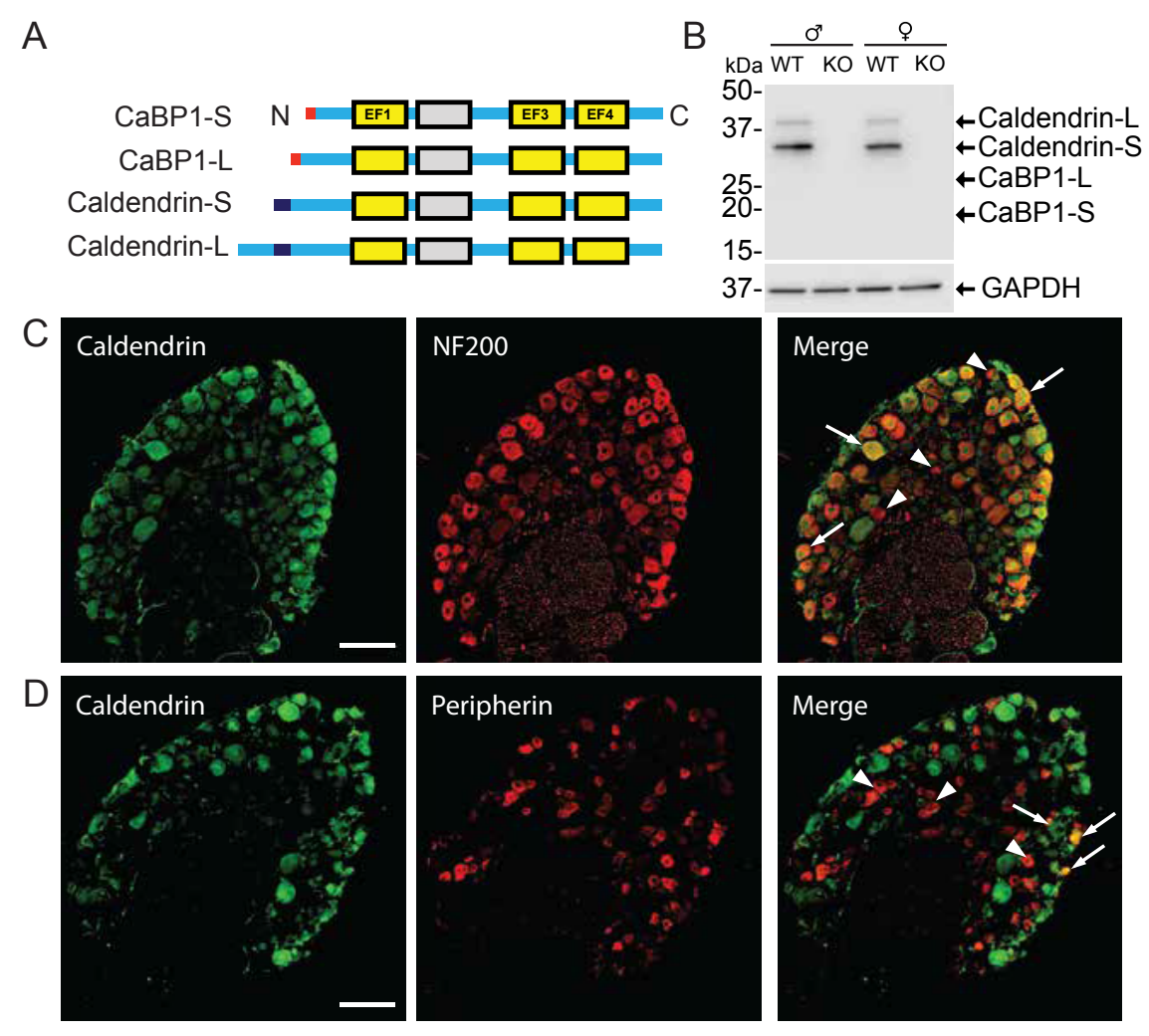

363 Figure 1. Caldendrin is the major CaBP1 variant in the DRG and is expressed primarily in myelinated DRGNs. (A) Schematic of CaBP1 splice variants containing EF-hand $\mathrm{Ca}^{2+}$ binding motifs and variable $\mathrm{N}$-terminal regions. CaBP1-S and CaBP1-L contain an N-myristoylation site (red). Caldendrin-S and Caldendrin- $L$ have a longer $N$-terminal region that includes a proline rich region (purple). (B) Western blots of DRG lysates from WT and KO mice probed with an antibody that recognizes all CaBP1 variants and ß-tubulin antibody for a protein loading control. 36- and 33- $\mathrm{kDa}$ bands corresponding to Caldendrin-S and Caldendrin- $\mathrm{L}$ were detected in WT but not in KO lysates. (C,D) Confocal micrographs from WT mouse DRG cryosections double-labeled with antibodies against caldendrin and NF200 or peripherin. In merged image, regions of colocalization are color-coded in yellow. Results are representative of 3 independent experiments. Scale bar, $100 \mu \mathrm{m}$. 
Figure 2
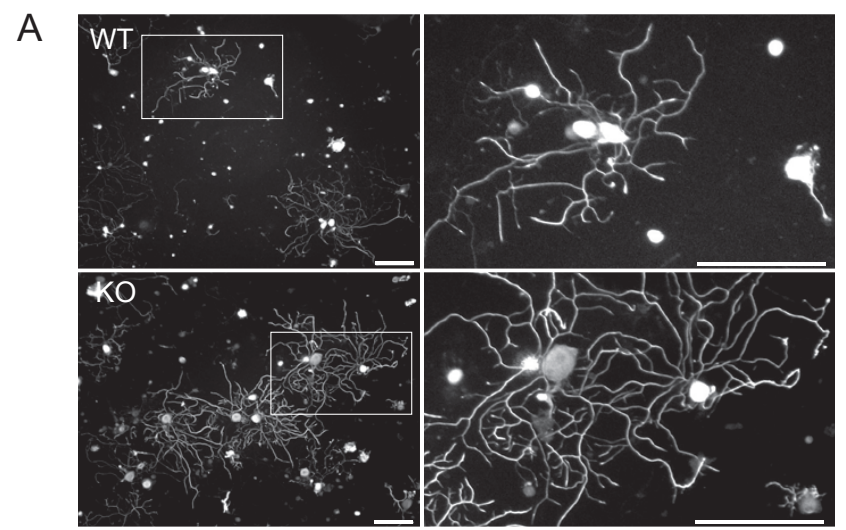

$\mathrm{B}$
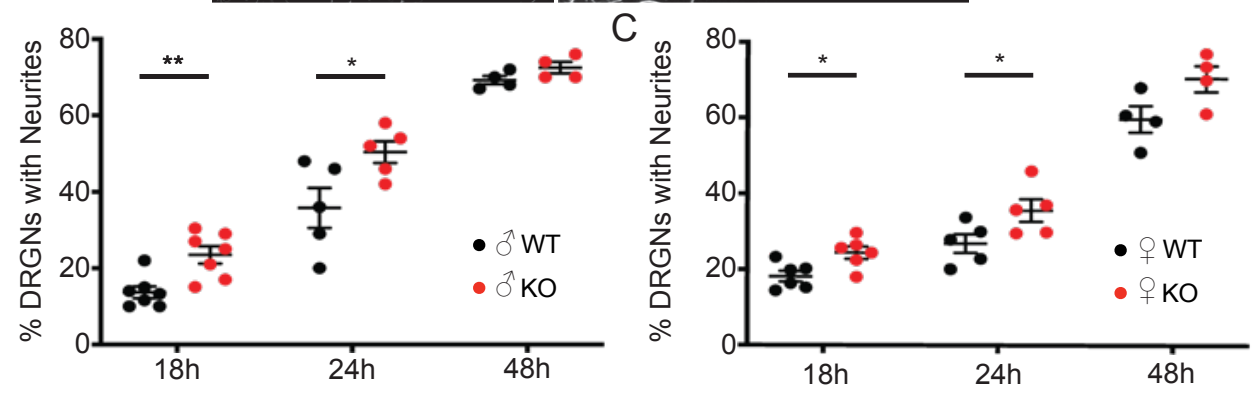

Figure 2. Neurite regeneration is enhanced in DRGNs of caldendrin KO than WT mice.

(A) Representative images of WT and KO DRGNs immunolabeled with NF200 antibodies after $24 \mathrm{~h}$ in culture. Boxed region in left panels is shown at high-magnification in right panels. Scale bars, $200 \mu \mathrm{m}$. (B,C) Percent of DRGNs with neurites measured as described in Materials and Methods for cultures from WT and KO males (B) and females (C) processed after the indicated times in culture. Each point represents the average obtained from a single culture $(n=300-400$ t-test. 
Figure 3
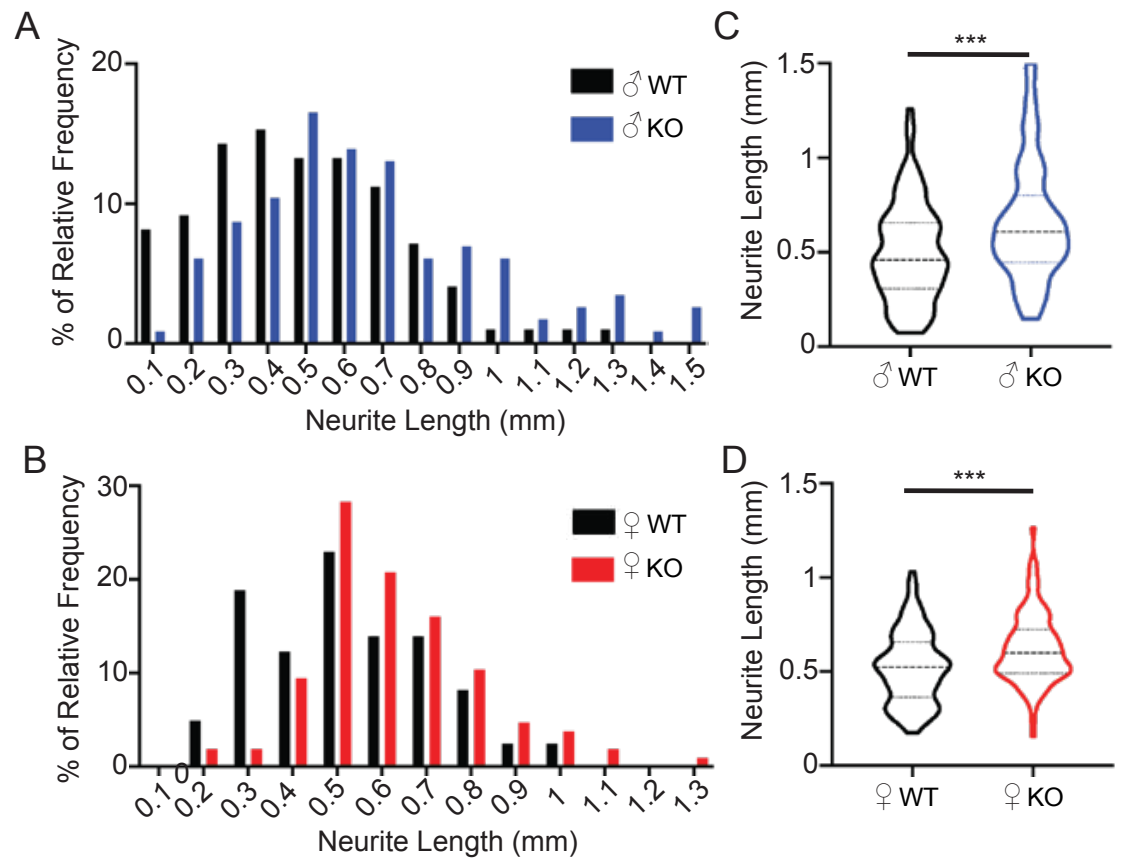

Figure 3. Caldendrin KO DRGNs exhibit enhanced neurite regrowth after in vitro axotomy. DRGNs were cultured for 3 days prior to replating and culture for $24 \mathrm{~h}$ prior to processing for NF200 immunofluorescence and measurement of the longest neurite per DRGN. (A,B) Frequency distributions of maximal neurite lengths in male (A) and female (B) WT and KO cultures. Data was collected from images of individual DRGNs ( $n=$ at least 32 images per culture, $n=3$ independent cultures). (C,D) Violin plots of data in $A, B$ with dashed and dotted lines representing the median and quartiles, respectively. ${ }^{* * *}, \mathrm{p}=0.0002$ by Mann-Whitney test. 


\section{Figure 4}

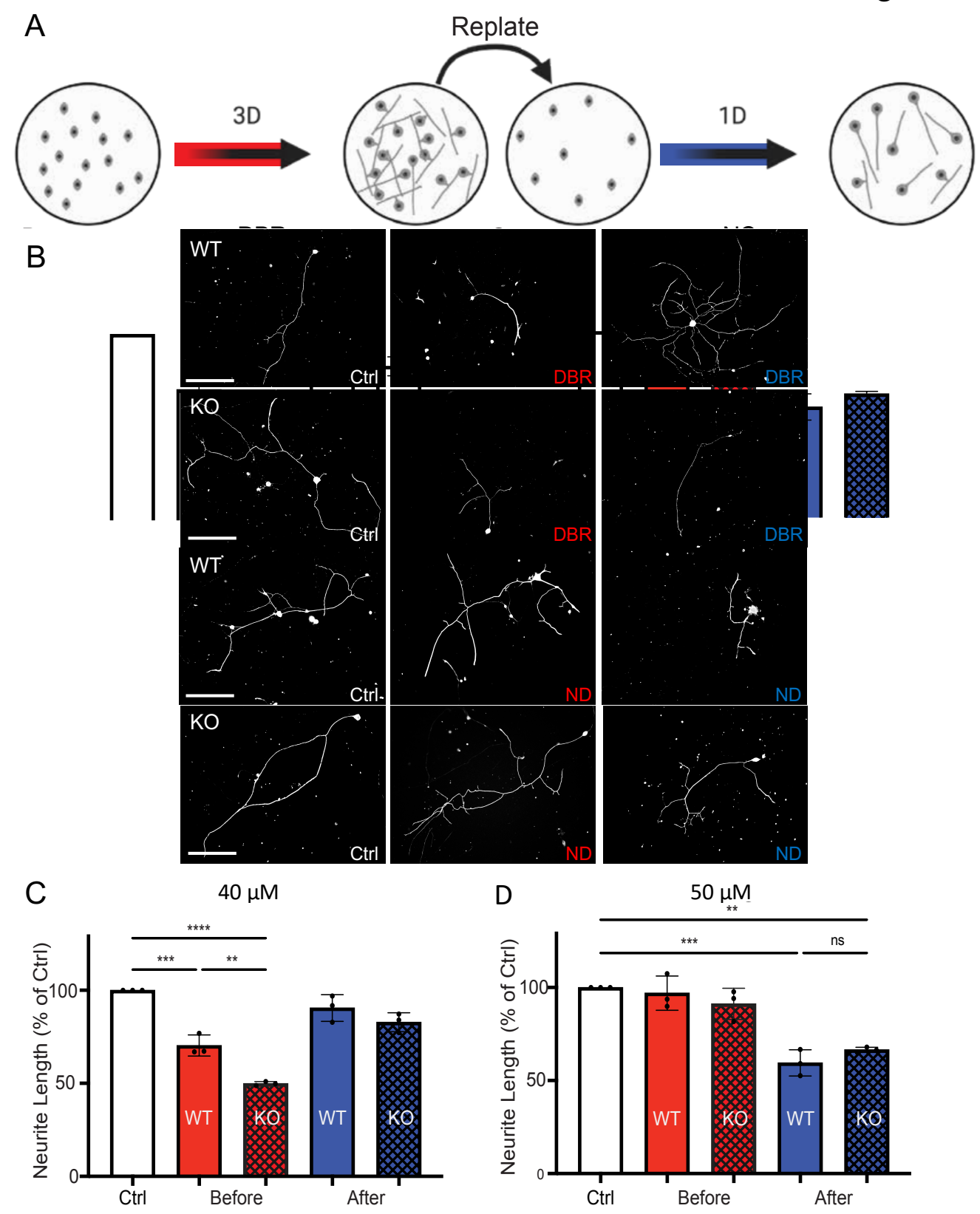

Figure 4. Caldendrin KO DRGNs exhibit stronger transcription-dependent neurite regeneration than WT DRGNs following in vitro axotomy. (A) Schematic of the experimental design for the same described in Fig.3. (B) Representative images of DRGNs from WT or KO male mice cultured with control medium (Ctrl) or medium containing 5,6-dichlorobenzimidazole riboside (DBR, $40 \mu \mathrm{M})$ or nocodazole (ND, $50 \mu \mathrm{M})$ before or after replating. Scale bars, $500 \mu \mathrm{m}$. $(C, D)$ Longest neurite was measured as in Fig. 3 and plotted for control and experimental groups. Each point represents the average obtained from $n=33$ DRGNs. Columns and error bars represent mean \pm SEM. ${ }^{* *} p<0.01,{ }^{* * *} p<0.001,{ }^{* * *} p<0.0001$, determined by two-way ANOVA, Tukey's multiple comparisons test. 
Figure 5

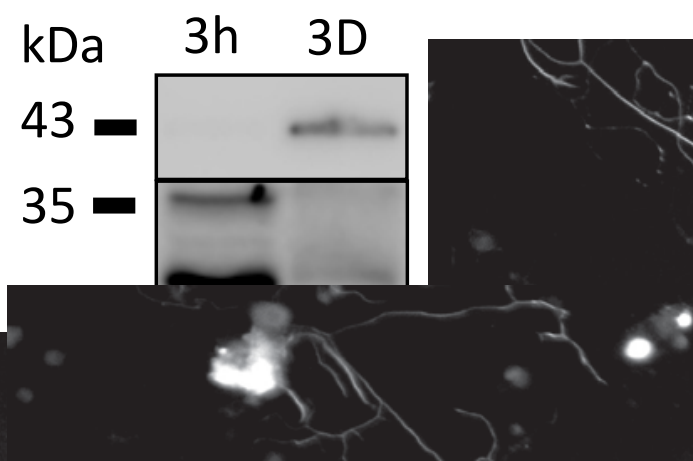

Figure 5. Caldendrin and GAP43 undergo opposing changes in expression with time in culture. DRGN cultures from WT male mice were lysed $3 \mathrm{~h}$ or $3 \mathrm{~d}$ after plating and subject to western blotting with antibodies against caldendrin, GAP43, and ß-Tubulin for loading control.

Figure 6

A

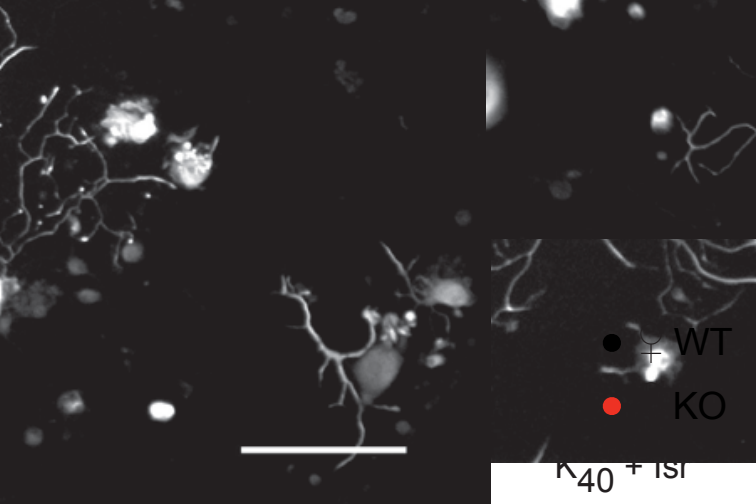

B

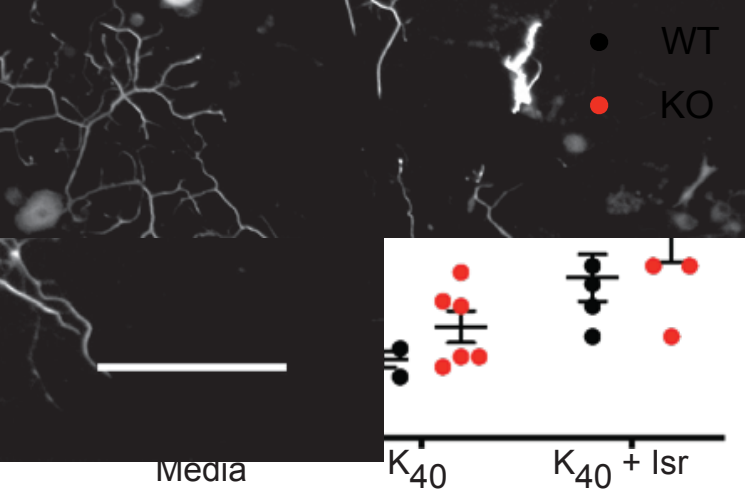

425

Figure 6. DRGNs from caldendrin $\mathrm{KO}$ females but not males are deficient in $\mathrm{Ca}_{\mathrm{v}} 1$ dependent neurite growth repression. $(A, B) D R G N s$ from female $(A)$ or male $(B)$ mice were cultured for $18 \mathrm{~h}$ in the presence of control medium (Ctrl) or medium containing $40 \mathrm{mM} \mathrm{KCl}\left(\mathrm{K}_{40}\right)$ \pm ISR $(10 \mu \mathrm{M})$ prior to determination of \% DRGNs with neurites as in Fig.2. Each point represents average determined from a total of $\sim 300$ DRGNs per culture. Bars represent mean \pm SEM. ${ }^{*} p<0.05,{ }^{* *} p<0.001$, by one-way ANOVA with Dunnett's multiple comparisons test. 


\section{Figure 7}
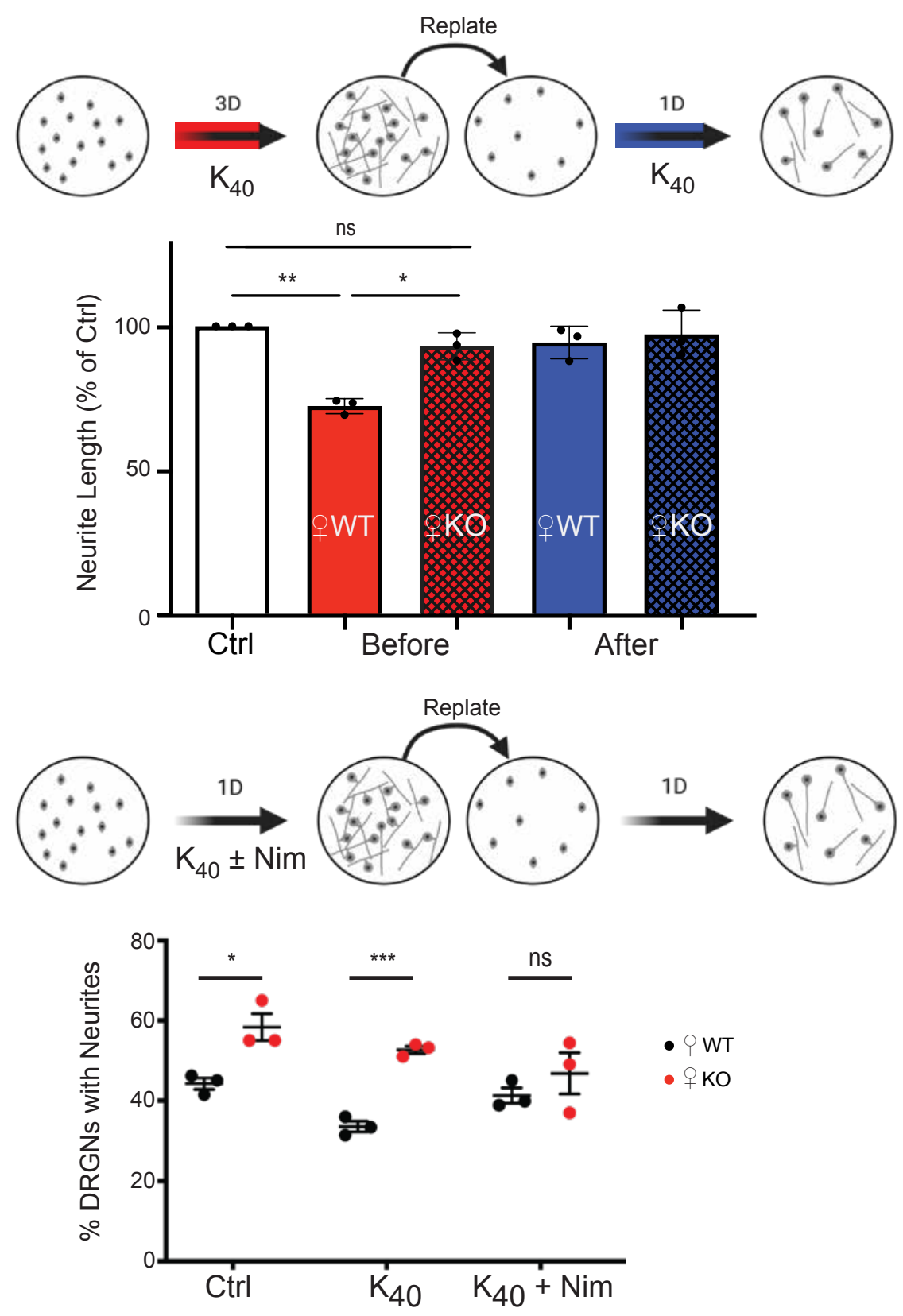

Figure 7. Coupling of $\mathrm{Ca}_{\mathrm{v}} 1$ channels to transcription-dependent repression of neurite regeneration following in vitro axotomy is disrupted in DRGNs from caldendrin KO females. (A) Schematic of the experimental design indicating exposure of cultures to $\mathrm{K}_{40}$ either before or after replating. (B) Longest neurite was measured as in Fig.3 and plotted for control and experimental groups in which WT or KO cultures were exposed to $\mathrm{K}_{40}$ before or after replating. ${ }^{*} p=0.01,{ }^{* *} p=0.002$ by two-way ANOVA, Tukey's multiple comparisons test. (C) Same as in A except that cultures were exposed to $\mathrm{K}_{40} \pm$ nimodipine $(\mathrm{Nim}, 10 \mu \mathrm{M})$ before or after replating. (D) $\%$ DRGNs with neurites was determined as in Fig.2, each data point represents the average determined from a total of 400 DRGNs per culture. ${ }^{*} p<0.05$, ${ }^{* * *} p=0.0003$, determined by t-test. 


\section{REFERENCES}

Boczek, T., Yu, Q., Zhu, Y., Dodge-Kafka, K. L., Goldberg, J. L., \& Kapiloff, M. S. (2021). cAMP at Perinuclear mAKAPalpha Signalosomes Is Regulated by Local $\mathrm{Ca}(2+)$ Signaling in Primary Hippocampal Neurons. eNeuro, 8(1). https://doi.org/10.1523/ENEURO.0298-20.2021

Chernov, A. V., Hullugundi, S. K., Eddinger, K. A., Dolkas, J., Remacle, A. G., Angert, M., James, B. P., Yaksh, T. L., Strongin, A. Y., \& Shubayev, V. I. (2020). A myelin basic protein fragment induces sexually dimorphic transcriptome signatures of neuropathic pain in mice. Journal of Biological Chemistry, 295(31), 10807-10821. https://doi.org/10.1074/ibc.RA120.013696

Chung, D., Shum, A., \& Caraveo, G. (2020). GAP-43 and BASP1 in Axon Regeneration: Implications for the Treatment of Neurodegenerative Diseases. Front Cell Dev Biol, 8, 567537. https://doi.org/10.3389/fcell.2020.567537

Costigan, M., Befort, K., Karchewski, L., Griffin, R. S., D'Urso, D., Allchorne, A., Sitarski, J., Mannion, J. W., Pratt, R. E., \& Woolf, C. J. (2002). Replicate high-density rat genome oligonucleotide microarrays reveal hundreds of regulated genes in the dorsal root ganglion after peripheral nerve injury. BMC Neurosci, 3, 16. https://doi.org/10.1186/1471-2202-3-16

Cui, G., Meyer, A. C., Calin-Jageman, I., Neef, J., Haeseleer, F., Moser, T., \& Lee, A. (2007). Ca2+binding proteins tune $\mathrm{Ca}^{2+}$ feedback to $\mathrm{Ca}_{\mathbf{v}} 1.3$ channels in auditory hair cells. The Journal of physiology, 585, 791-803. 17947313

Dieterich, D. C., Karpova, A., Mikhaylova, M., Zdobnova, I., Konig, I., Landwehr, M., Kreutz, M., Smalla, K. H., Richter, K., Landgraf, P., Reissner, C., Boeckers, T. M., Zuschratter, W., Spilker, C., Seidenbecher, C. I., Garner, C. C., Gundelfinger, E. D., \& Kreutz, M. R. (2008). Caldendrin-Jacob: a protein liaison that couples NMDA receptor signalling to the nucleus. PLoS Biol, 6(2), e34. https://doi.org/10.1371/journal.pbio.0060034

Enes, J., Langwieser, N., Ruschel, J., Carballosa-Gonzalez, M. M., Klug, A., Traut, M. H., Ylera, B., Tahirovic, S., Hofmann, F., Stein, V., Moosmang, S., Hentall, I. D., \& Bradke, F. (2010). Electrical activity suppresses axon growth through $\mathrm{Ca}(\mathrm{v}) 1.2$ channels in adult primary sensory neurons [Research Support, Non-U.S. Gov't]. Current biology : CB, 20(13), 11541164. https://doi.org/10.1016/j.cub.2010.05.055

Fields, R. D., Neale, E. A., \& Nelson, P. G. (1990). Effects of patterned electrical activity on neurite outgrowth from mouse sensory neurons. The Journal of Neuroscience, 10(9), 2950-2964. https://www.ncbi.nlm.nih.gov/pubmed/2398369

Franco-Enzastiga, U., Garcia, G., Murbartian, J., Gonzalez-Barrios, R., Salinas-Abarca, A. B., Sanchez-Hernandez, B., Tavares-Ferreira, D., Herrera, L. A., Barragan-Iglesias, P., DelgadoLezama, R., Price, T. J., \& Granados-Soto, V. (2021). Sex-dependent pronociceptive role of spinal alpha5 -GABAA receptor and its epigenetic regulation in neuropathic rodents. $J$ Neurochem, 156(6), 897-916. https://doi.org/10.1111/jnc.15140

Goldberg, J. L., Espinosa, J. S., Xu, Y., Davidson, N., Kovacs, G. T., \& Barres, B. A. (2002). Retinal ganglion cells do not extend axons by default: promotion by neurotrophic signaling and electrical activity. Neuron, 33(5),

689-702. https://www.ncbi.nlm.nih.gov/pubmed/11879647 
Gomez, T. M., \& Zheng, J. Q. (2006). The molecular basis for calcium-dependent axon pathfinding. Nature reviews. Neuroscience, 7(2), 115-125. https://doi.org/10.1038/nrn1844

Gorny, X., Mikhaylova, M., Seeger, C., Reddy, P. P., Reissner, C., Schott, B. H., Helena Danielson, U., Kreutz, M. R., \& Seidenbecher, C. (2012). AKAP79/150 interacts with the neuronal calcium-binding protein caldendrin [In Vitro

Research Support, Non-U.S. Gov't]. J Neurochem, 122(4), 714-726. https://doi.org/10.1111/j.1471-4159.2012.07828.x

Haeseleer, F., Imanishi, Y., Maeda, T., Possin, D. E., Maeda, A., Lee, A., Rieke, F., \& Palczewski, K. (2004). Essential role of $\mathrm{Ca}^{2+}$-binding protein 4, a Cav1.4 channel regulator, in photoreceptor synaptic function. Nature Neuroscience, 7(10), 1079-1087. 15452577

Haeseleer, F., Sokal, I., Verlinde, C. L., Erdjument-Bromage, H., Tempst, P., Pronin, A. N., Benovic, J. L., Fariss, R. N., \& Palczewski, K. (2000). Five members of a novel Ca ${ }^{2+}$-binding protein (CABP) subfamily with similarity to calmodulin. Journal of Biological Chemistry, 275(2), 1247-1260. 10625670

Haynes, L. P., Tepikin, A. V., \& Burgoyne, R. D. (2004). Calcium-binding protein 1 is an inhibitor of agonist-evoked, inositol 1,4,5-trisphosphate-mediated calcium signaling. Journal of Biological Chemistry, 279(1), 547-555. 14570872

Henley, J., \& Poo, M. M. (2004). Guiding neuronal growth cones using Ca2+ signals. Trends Cell Biol, 14(6), 320-330. https://doi.org/10.1016/j.tcb.2004.04.006

Huebner, E. A., Budel, S., Jiang, Z., Omura, T., Ho, T. S., Barrett, L., Merkel, J. S., Pereira, L. M., Andrews, N. A., Wang, X., Singh, B., Kapur, K., Costigan, M., Strittmatter, S. M., \& Woolf, C. J. (2019). Diltiazem Promotes Regenerative Axon Growth. Mol Neurobiol, 56(6), 39483957. https://doi.org/10.1007/s12035-018-1349-5

Huebner, E. A., \& Strittmatter, S. M. (2009). Axon regeneration in the peripheral and central nervous systems. Results Probl Cell Differ, 48, 339-351. https://doi.org/10.1007/400_2009_19

Juarez, I., Morales-Medina, J. C., Flores-Tochihuitl, J., Juarez, G. S., Flores, G., \& Oseki, H. C. (2019). Tooth pulp injury induces sex-dependent neuronal reshaping in the ventral posterolateral nucleus of the rat thalamus. J Chem Neuroanat, 96, 16-21. https://doi.org/10.1016/i.jchemneu.2018.10.007

Kasri, N. N., Holmes, A. M., Bultynck, G., Parys, J. B., Bootman, M. D., Rietdorf, K., Missiaen, L., McDonald, F., De Smedt, H., Conway, S. J., Holmes, A. B., Berridge, M. J., \& Roderick, H. L. (2004). Regulation of InsP3 receptor activity by neuronal $\mathrm{Ca}^{2+-b i n d i n g}$ proteins. The EMBO journal, 23(2), 312-321. 14685260

Kim, K. Y., Scholl, E. S., Liu, X., Shepherd, A., Haeseleer, F., \& Lee, A. (2014). Localization and expression of CaBP1/caldendrin in the mouse brain. Neuroscience, 268, 33-47. https://doi.org/10.1016/j.neuroscience.2014.02.052

Kubo, Y., Baba, K., Toriyama, M., Minegishi, T., Sugiura, T., Kozawa, S., Ikeda, K., \& Inagaki, N. (2015). Shootin1-cortactin interaction mediates signal-force transduction for axon outgrowth. The Journal of cell biology, 210(4), 663-676. https://doi.org/10.1083/jcb.201505011

Lai, Y. J., Zhu, B. L., Sun, F., Luo, D., Ma, Y. L., Luo, B., Tang, J., Xiong, M. J., Liu, L., Long, Y., Hu, X. T., He, L., Deng, X. J., Zhang, J. H., Yang, J., Yan, Z., \& Chen, G. J. (2019). Estrogen receptor 
alpha promotes Cav1.2 ubiquitination and degradation in neuronal cells and in APP/PS1 mice. Aging Cell, 18(4), e12961. https://doi.org/10.1111/acel.12961

Laube, G., Seidenbecher, C. I., Richter, K., Dieterich, D. C., Hoffmann, B., Landwehr, M., Smalla, K. H., Winter, C., Bockers, T. M., Wolf, G., Gundelfinger, E. D., \& Kreutz, M. R. (2002). The neuron-specific $\mathrm{Ca}^{2+-b i n d i n g}$ protein caldendrin: gene structure, splice isoforms, and expression in the rat central nervous system. Mol. Cell. Neurosci., 19(3), 459-475. 11906216

Lawson, S. N., \& Waddell, P. J. (1991). Soma neurofilament immunoreactivity is related to cell size and fibre conduction velocity in rat primary sensory neurons. J Physiol, 435, 41-63. https://doi.org/10.1113/jphysiol.1991.sp018497

Lin, Y. T., \& Chen, J. C. (2018). Dorsal Root Ganglia Isolation and Primary Culture to Study Neurotransmitter Release. Journal of visualized experiments : JoVE(140). https://doi.org/10.3791/57569

Mecklenburg, J., Zou, Y., Wangzhou, A., Garcia, D., Lai, Z., Tumanov, A. V., Dussor, G., Price, T. J., \& Akopian, A. N. (2020). Transcriptomic sex differences in sensory neuronal populations of mice. Scientific reports, 10(1), 15278. https://doi.org/10.1038/s41598-020-72285-z

Mikhaylova, M., Bar, J., van Bommel, B., Schatzle, P., YuanXiang, P., Raman, R., Hradsky, J., Konietzny, A., Loktionov, E. Y., Reddy, P. P., Lopez-Rojas, J., Spilker, C., Kobler, O., Raza, S. A., Stork, O., Hoogenraad, C. C., \& Kreutz, M. R. (2018). Caldendrin Directly Couples Postsynaptic Calcium Signals to Actin Remodeling in Dendritic Spines. Neuron, 97(5), 1110-1125 e1114. https://doi.org/10.1016/j.neuron.2018.01.046

Moskowitz, P. F., Smith, R., Pickett, J., Frankfurter, A., \& Oblinger, M. M. (1993). Expression of the class III beta-tubulin gene during axonal regeneration of rat dorsal root ganglion neurons. Journal of neuroscience research, 34(1), 129-134. https://doi.org/10.1002/jnr.490340113

Oh, Y. M., Mahar, M., Ewan, E. E., Leahy, K. M., Zhao, G., \& Cavalli, V. (2018). Epigenetic regulator UHRF1 inactivates REST and growth suppressor gene expression via DNA methylation to promote axon regeneration. Proc Natl Acad Sci U S A, 115(52), E12417-E12426. https://doi.org/10.1073/pnas.1812518115

Popko, J., Fernandes, A., Brites, D., \& Lanier, L. M. (2009). Automated analysis of NeuronJ tracing data. Cytometry A, 75(4), 371-376. https://doi.org/10.1002/cyto.a. 20660

Robson, S. J., \& Burgoyne, R. D. (1989). L-type calcium channels in the regulation of neurite outgrowth from rat dorsal root ganglion neurons in culture. Neurosci Lett, 104(1-2), 110114. https://www.ncbi.nlm.nih.gov/pubmed/2554216

Saijilafu, Hur, E. M., Liu, C. M., Jiao, Z., Xu, W. L., \& Zhou, F. Q. (2013). PI3K-GSK3 signalling regulates mammalian axon regeneration by inducing the expression of Smad1. Nat Commun, 4, 2690. https://doi.org/10.1038/ncomms3690

Sarkar, S. N., Huang, R. Q., Logan, S. M., Yi, K. D., Dillon, G. H., \& Simpkins, J. W. (2008). Estrogens directly potentiate neuronal L-type Ca2+ channels. Proc Natl Acad Sci U S A, 105(39), 15148-15153. https://doi.org/10.1073/pnas.0802379105

Scheib, J., \& Hoke, A. (2013). Advances in peripheral nerve regeneration. Nat Rev Neurol, 9(12), 668-676. https://doi.org/10.1038/nrneurol.2013.227

Schreyer, D. J., \& Skene, J. H. (1991). Fate of GAP-43 in ascending spinal axons of DRG neurons after peripheral nerve injury: delayed accumulation and correlation with regenerative 
potential. The Journal of Neuroscience, 11(12), 3738-3751.

Seidenbecher, C. I., Langnaese, K., Sanmarti-Vila, L., Boeckers, T. M., Smalla, K. H., Sabel, B. A., calcium-binding protein confined to the somato-dendritic compartment. Journal of Biological Chemistry, 273(33), 21324-21331. 9694893

Singh, K. K., \& Miller, F. D. (2005). Activity regulates positive and negative neurotrophin-derived signals to determine axon competition. Neuron, 45(6), 837-845. https://doi.org/10.1016/j.neuron.2005.01.049

Smith, D. S., \& Skene, J. H. (1997). A transcription-dependent switch controls competence of adult neurons for distinct modes of axon growth. J Neurosci, 17(2), 646-658. https://www.ncbi.nlm.nih.gov/pubmed/8987787

Takei, K., Shin, R. M., Inoue, T., Kato, K., \& Mikoshiba, K. (1998). Regulation of nerve growth mediated by inositol 1,4,5-trisphosphate receptors in growth cones. Science, 282(5394), 1705-1708. https://doi.org/10.1126/science.282.5394.1705

Tippens, A. L., \& Lee, A. (2007). Caldendrin: a neuron-specific modulator of Cav1.2 (L-type) Ca2+ channels. Journal of Biological Chemistry, 282, 8464-8473. 17224447

Vega-Vela, N. E., Osorio, D., Avila-Rodriguez, M., Gonzalez, J., Garcia-Segura, L. M., Echeverria, V., \& Barreto, G. E. (2017). L-Type Calcium Channels Modulation by Estradiol. Mol Neurobiol, 54(7), 4996-5007. https://doi.org/10.1007/s12035-016-0045-6

Wu, H., Petitpre, C., Fontanet, P., Sharma, A., Bellardita, C., Quadros, R. M., Jannig, P. R., Wang, Y., Heimel, J. A., Cheung, K. K. Y., Wanderoy, S., Xuan, Y., Meletis, K., Ruas, J., Gurumurthy, C. B., Kiehn, O., Hadjab, S., \& Lallemend, F. (2021). Distinct subtypes of proprioceptive dorsal root ganglion neurons regulate adaptive proprioception in mice. Nature communications, 12(1), 1026. https://doi.org/10.1038/s41467-021-21173-9

Wu, J., \& Xie, X. (2006). Comparative sequence analysis reveals an intricate network among REST, CREB and miRNA in mediating neuronal gene expression. Genome Biol, 7(9), R85. https://doi.org/10.1186/gb-2006-7-9-r85

Yang, T., Choi, J. E., Soh, D., Tobin, K., Joiner, M. L., Hansen, M., \& Lee, A. (2018). CaBP1 regulates Cav1 L-type $\mathrm{Ca}(2+)$ channels and their coupling to neurite growth and gene transcription in mouse spiral ganglion neurons. Mol Cell Neurosci, 88, 342-352. https://doi.org/10.1016/j.mcn.2018.03.005

Yang, T., Scholl, E. S., Pan, N., Fritzsch, B., Haeseleer, F., \& Lee, A. (2016). Expression and Localization of CaBP Ca2+ Binding Proteins in the Mouse Cochlea. PLoS One, 11(1), e0147495. https://doi.org/10.1371/journal.pone.0147495

Yang, X., Mao, X., Xu, G., Xing, S., Chattopadhyay, A., Jin, S., \& Salama, G. (2018). Estradiol upregulates L-type $\mathrm{Ca}(2+)$ channels via membrane-bound estrogen receptor/phosphoinositide-3-kinase/Akt/cAMP response element-binding protein signaling pathway. Heart Rhythm, 15(5), 741-749. https://doi.org/10.1016/i.hrthm.2018.01.019

Yiera, B., \& Bradke, F. (2006). Stimulating Intrinsic Growth Potential in Mammalian Neurons. In C. G. Becker \& T. Becker (Eds.), Model Organisms in Spinal Cord Regeneration Wiley-VCH Verlag GmbH \& Co. KGaA. 
615 Zheng, Y., Liu, P., Bai, L., Trimmer, J. S., Bean, B. P., \& Ginty, D. D. (2019). Deep Sequencing of Somatosensory Neurons Reveals Molecular Determinants of Intrinsic Physiological Properties. Neuron, 103(4), 598-616 e597. https://doi.org/10.1016/j.neuron.2019.05.039

618 Zhou, H., Kim, S. A., Kirk, E. A., Tippens, A. L., Sun, H., Haeseleer, F., \& Lee, A. (2004). Ca ${ }^{2+}$-binding protein-1 facilitates and forms a postsynaptic complex with Cav1.2 (L-type) $\mathrm{Ca}^{2+}$ channels. The Journal of Neuroscience, 24(19), 4698-4708. 15140941

621 Zhou, H., Yu, K., McCoy, K. L., \& Lee, A. (2005). Molecular mechanism for divergent regulation of $\mathrm{Ca}_{\sqrt{ }} 1.2 \mathrm{Ca}^{2+}$ channels by calmodulin and $\mathrm{Ca}^{2+-b i n d i n g ~ p r o t e i n-1 . ~ J o u r n a l ~ o f ~ B i o l o g i c a l ~}$ Chemistry, 280(33), 29612-29619. 15980432 